DESY 98-023

ISSN 0418-9833

MPI/PhT/98-020

hep-ph/9803256

March 1998

\title{
TEVATRON-HERA Colour-Octet Charmonium Anomaly Versus Higher-Order QCD Effects
}

\author{
Bernd A. Kniehl ${ }^{1}$ And Gustav Kramer ${ }^{2}$ \\ ${ }^{1}$ Max-Planck-Institut für Physik (Werner-Heisenberg-Institut), \\ Föhringer Ring 6, 80805 Munich, Germany \\ 2 II. Institut für Theoretische Physik*, Universität Hamburg, \\ Luruper Chaussee 149, 22761 Hamburg, Germany
}

\begin{abstract}
Approximately taking into account the higher-order effects due to multiple-gluon initial-state radiation, we extract from the latest Tevatron data of prompt $J / \psi$ hadroproduction the leading colour-octet matrix elements within the nonrelativisticQCD (NRQCD) factorization formalism proposed by Bodwin, Braaten, and Lepage. We find that the matrix elements which describe the formation of $J / \psi$ mesons from colour-octet $c \bar{c}$ pairs in the angular-momentum states ${ }^{2 S+1} L_{J}={ }^{1} S_{0}$ and ${ }^{3} P_{J}$, with $J=0,1,2$, which are responsible for the excess of the predicted cross section of inelastic $J / \psi$ photoproduction over the existing HERA data at high values of the inelasticity variable $z$, are significantly reduced. We conclude that it is premature to proclaim a discrepancy between the Tevatron and HERA measurements of inclusive $J / \psi$ production in the NRQCD framework. We also consider $J / \psi$ mesons originating from the radiative feed down of promptly produced $\chi_{c} J$ mesons.
\end{abstract}

PACS numbers: 13.60.-r, 13.85.-t, 13.85.Ni, 14.40.Lb

\footnotetext{
*Supported by Bundesministerium für Forschung und Technologie, Bonn, Germany, under Contract $057 \mathrm{HH}$ 92P (5), and by EU Program Human Capital and Mobility through Network Physics at High Energy Colliders under Contract CHRX-CT93-0357 (DG12 COMA).
} 


\section{Introduction}

Since its discovery in 1974, the $J / \psi$ meson has provided a useful laboratory for quantitatively testing quantum chromodynamics (QCD) and, in particular, the interplay of perturbative and nonperturbative phenomena. Recently, the cross section of inclusive $J / \psi$ hadroproduction measured in $p \bar{p}$ collisions at the Fermilab Tevatron [1,2] turned out to be more than one order of magnitude in excess of what used to be the best theoretical prediction [3], based on the colour-singlet model (CSM). As a solution to this puzzle, Bodwin, Braaten, and Lepage [4] proposed the existence of so-called colour-octet processes to fill the gap. The central idea is that $c \bar{c}$ pairs are produced at short distances in colour-octet states and subsequently evolve into physical (colour-singlet) charmonia by the nonperturbative emission of soft gluons. The underlying theoretical framework is provided by nonrelativistic QCD (NRQCD) endowed with a particular factorization hypothesis, which implies a separation of short-distance coefficients, which are amenable to perturbative QCD, from long-distance matrix elements, which must be extracted from experiment. This formalism involves a double expansion in the strong coupling constant $\alpha_{s}$ and the relative velocity $v$ of the bound $c$ quarks, and takes the complete structure of the charmonium Fock space into account.

In order to convincingly establish the phenomenological significance of the colour-octet mechanism, it is indispensable to identify it in other kinds of high-energy experiments as well. In the meantime, there have been a number of interesting proposals and encouraging results concerning possible colour-octet signatures of inclusive $J / \psi$ production in $e^{+} e^{-}$ annihilation in the continuum [5] and on the $Z$-boson resonance [6], hadronic collisions in the fixed-target mode [7], $B$-meson decays [8], and photoproduction in the forward direction [9] and at finite transverse momentum [10,11]. Although the experimental Zboson-decay data from CERN LEP [12] are in agreement with the predicted colour-octet signature, they do not exclude the hypothesis that the observed prompt charmonium signal is produced by colour-singlet processes alone.

In the case of inelastic $J / \psi$ photoproduction, however, NRQCD with colour-octet matrix elements tuned [13] to fit the Tevatron data [1] predicts [10,11] at leading order (LO) a distinct rise in cross section as $z \rightarrow 1$, where $z$ is the fraction of the photon energy transferred to the $J / \psi$ meson in the proton rest frame, which is not observed by the H1 [14] and ZEUS [15] collaborations at DESY HERA. This colour-octet charmonium anomaly has cast doubts on the validity of the NRQCD factorization hypothesis [4], which seems so indispensible to interpret the Tevatron data in a meaningful way.

This paper is an attempt to rescue the NRQCD approach by approximately taking into account dominant higher-order (HO) QCD effects. The basic idea is as follows. The predicted excess over the HERA data at $z$ close to unity is chiefly generated by colouroctet $c \bar{c}$ pairs in the states ${ }^{1} S_{0},{ }^{3} P_{0}$, and ${ }^{3} P_{2}$ [10,11], where we use the spectroscopic notation ${ }^{2 S+1} L_{J}$ to indicate the spin $S$, the orbital angular momentum $L$, and the total angular momentum $J$. On the other hand, in hadroproduction at the Tevatron, the contributions from the colour-octet ${ }^{1} S_{0}$ and ${ }^{3} P_{J}$ states fall off much more strongly with increasing $p_{T}$ than the one due to the colour-octet ${ }^{3} S_{1}$ state [13], which is greatly suppressed in the 
quasi-elastic limit of photoproduction [10,11]. Consequently, the nonperturbative matrix elements which are responsible for the colour-octet charmonium crisis are essentially fixed by the Tevatron data in the low- $p_{T}$ regime. This is precisely where the LO approximation used in Ref. [13 is expected to become unreliable due to multiple-gluon radiation from the initial and final states. In Ref. [16], this phenomenon was carefully analyzed in a Monte Carlo framework and found to significantly increase the LO cross section. In this paper, we perform fits to the latest prompt $J / \psi$ data taken by the CDF collaboration [2] at the Tevatron incorporating this information [16 on the dominant HO QCD effects. We then update the NRQCD predictions for inelastic $J / \psi$ photoproduction at HERA to find out whether the colour-octet charmonium anomaly persists. We also carry out a LO analysis for $J / \psi$ mesons originating from the radiative feed down of promptly produced $\chi_{c J}$ mesons.

This paper is organized as follows. In Section 2, we specify the theoretical input for our analysis. In Section 3, we describe our fitting procedure and present our results for the various $J / \psi$ and $\chi_{c J}$ matrix elements. In Section 4 , we present the resulting predictions for inelastic $J / \psi$ and $\chi_{c J}$ photoproduction at HERA. Our conclusions are summarized in Section 5 .

\section{Theoretical input}

We now describe the theoretical framework for our analysis. We calculate the cross section for the inclusive production $A+B \rightarrow H+X$ of the physical charmonium state $H$ in the collision of two hadrons, $A$ and $B$, in the parton model of QCD, i.e. $A$ and $B$ are represented by their parton density functions (PDF's). In the case of photoproduction, $A$ is a quasi-real photon, which either directly interacts with the partons inside hadron $B$ (direct photoproduction) or fluctuates into a bunch of quarks and gluons, which in turn interact with the partons inside hadron $B$, while the photon remnants give rise to hadronic activity in the original photon flight direction (resolved photoproduction). Depending on the transverse momentum $p_{T}$ of the $H$ meson, we adopt two different pictures to describe its production. For $p_{T}$ values of the order of the charmonium mass $M_{H} \approx 2 m_{c}$, we consider the formation of a collinear $c \bar{c}$ pair within the primary hardscattering process (fusion). In the limit $p_{T} \gg M_{H}$, only those subprocesses survive where the $c \bar{c}$ pair is created from a single high-energy gluon, charm quark or antiquark which is close to its mass shell (fragmentation). It is then useful to describe this two-step process as a convolution of the hard-scattering cross section for single-parton production with an appropriate fragmentation function $(\mathrm{FF})$. In both cases, the factorization theorem of NRQCD [4] allows for a systematical treatment of the transition from the $c \bar{c}$ pair, in state $n$, to the physical $H$ meson. The states $n=\left[\underline{1},{ }^{2 S+1} L_{J}\right],\left[\underline{8},{ }^{2 S+1} L_{J}\right]$, where $\underline{1}$ and $\underline{8}$ indicate the colour multiplicity, span the whole $c \bar{c}$ Fock space. This formalism comprises a separation of short-distance parts, which are amenable to perturbative QCD, from longdistance matrix elements $\left\langle\mathcal{O}^{H}[n]\right\rangle$, which must be extracted from experiment. In the case 
of fusion, the differential cross section of $i j \rightarrow H+X$ is decomposed as

$$
\frac{d \sigma}{d t}(i j \rightarrow H+X)=\sum_{n} \frac{d \sigma}{d t}(i j \rightarrow c \bar{c}[n]+k)\left\langle\mathcal{O}^{H}[n]\right\rangle,
$$

where the cross sections of the partonic subprocess $i j \rightarrow c \bar{c}[n]+k$ may be calculated in NRQCD as a perturbation expansion in the strong coupling constant $\alpha_{s}(\mu)$, since the renormalization scale $\mu$ is typically set by the transverse mass $m_{T}=\sqrt{4 m_{c}^{2}+p_{T}^{2}}$ of the $H$ meson. In the case of fragmentation, one has

$$
D_{i \rightarrow H}(x, \mu)=\sum_{n} d_{i \rightarrow n}(x, \mu)\left\langle\mathcal{O}^{H}[n]\right\rangle,
$$

where $d_{i \rightarrow n}(x, \mu)$ gives the probability for the parton $i$ to form a jet that includes a $c \bar{c}$ pair in state $n$ carrying the longitudinal-momentum fraction $x$. The coefficient $d_{i \rightarrow n}\left(x, \mu_{0}\right)$ at the initial scale $\mu_{0}=2 m_{c}$ involves only momenta of order $m_{c}$, and can thus be calculated within NRQCD in powers of $\alpha_{s}\left(\mu_{0}\right)$. The evolution of the FF $D_{i \rightarrow H}\left(x, \mu_{0}\right)$ up to higher fragmentation scales $\mu=M_{T}$ is ruled by the timelike Altarelli-Parisi (AP) equations, which may be conveniently solved in $x$ space 17 .

The relative importance of the various terms in Eqs. (11) and (2) may be estimated my means of NRQCD velocity scaling rules 4 . In the limit $v \rightarrow 0$, where $v$ is the average velocity of the charm quark in the $H$-meson rest frame, each of the nonperturbative matrix elements $\left\langle\mathcal{O}^{H}[n]\right\rangle$ scales with a definite power of $v$. Thus, Eqs. (11) and (2) can be organized as a double expansion in $\alpha_{s}$ and $v$. At leading order in $v$, Eqs. (1) and (2) reduce to the standard factorization formulas of the colour-singlet model, which contain just the term referring to the state $n$ of the physical $H$ meson, i.e. $\left[\underline{1},{ }^{3} S_{1}\right]$ and $\left[\underline{1},{ }^{3} P_{J}\right]$ for the $J / \psi$ and $\chi_{c J}$ mesons, respectively. In the case of the $J / \psi$ meson, $\left\langle\mathcal{O}^{J / \psi}\left[\underline{1},{ }^{3} S_{1}\right]\right\rangle$ is related to the nonrelativisitic radial wave function at the origin, $R_{J / \psi}(0)$, and may thus be extracted from the measured leptonic annihilation rate. The QCD-improved [18] relation reads

$$
\Gamma\left(J / \psi \rightarrow \ell^{+} \ell^{-}\right)=\frac{8 \pi \alpha^{2} e_{c}^{2}}{9 M_{J / \psi}^{2}}\left\langle\mathcal{O}^{J / \psi}\left[\underline{1},{ }^{3} S_{1}\right]\right\rangle\left(1-\frac{16}{3} \frac{\alpha_{s}\left(M_{J / \psi}\right)}{\pi}\right),
$$

where $\alpha$ is Sommerfeld's fine-structure constant and $e_{c}=2 / 3$ is the fractional charge of the charm quark. In a similar way, $\left\langle\mathcal{O}^{\chi_{c J}}\left[\underline{1},{ }^{3} P_{J}\right]\right\rangle$ may be determined from the measured partial widths of the $\chi_{c J}$-meson decays into light hadrons [19] or two photons [20]. The leading colour-octet matrix elements of the $J / \psi$ meson are $\left\langle\mathcal{O}^{J / \psi}\left[\underline{8},{ }^{1} S_{0}\right]\right\rangle,\left\langle\mathcal{O}^{J / \psi}\left[\underline{8},{ }^{3} S_{1}\right]\right\rangle$, and $\left\langle\mathcal{O}^{J / \psi}\left[\underline{8},{ }^{3} P_{J}\right]\right\rangle$, with $J=0,1,2$. The leading colour-octet matrix element of the $\chi_{c J}$ meson is $\left\langle\mathcal{O}^{\chi_{c J}}\left[\underline{8},{ }^{3} S_{1}\right]\right\rangle$. Due to heavy-quark spin symmetry, the $J$-dependent matrix elements satisfy the multiplicity relations

$$
\begin{aligned}
\left\langle\mathcal{O}^{J / \psi}\left[\underline{8},{ }^{3} P_{J}\right]\right\rangle & =(2 J+1)\left\langle\mathcal{O}^{J / \psi}\left[\underline{8},{ }^{3} P_{0}\right]\right\rangle, \\
\left\langle\mathcal{O}^{\chi_{c J}}\left[\underline{1},{ }^{3} P_{J}\right]\right\rangle & =(2 J+1)\left\langle\mathcal{O}^{\chi_{c 0}}\left[\underline{1},{ }^{3} P_{0}\right]\right\rangle, \\
\left\langle\mathcal{O}^{\chi_{c J}}\left[\underline{8},{ }^{3} S_{1}\right]\right\rangle & =(2 J+1)\left\langle\mathcal{O}^{\chi_{c 0}}\left[\underline{8},{ }^{3} S_{1}\right]\right\rangle,
\end{aligned}
$$


up to terms of relative order $v^{2}$. The scaling with $v$ (and $m_{c}$ ) of the various $J / \psi$ and $\chi_{c J}$ matrix elements is indicated in Tables 11 and 2, respectively.

The leading colour-octet matrix elements for the $J / \psi$ and $\chi_{c J}$ mesons have been determined through fits, based on the fusion [13,21] and fragmentation [22] mechanisms at $\mathrm{LO}$, to CDF data on inclusive charmonium hadroproduction [1,2]. In this paper, we perform new fits to the most recent CDF data [2] taking into account information on the HO QCD effects induced by multiple-gluon radiation in a Monte Carlo framework [16] and consistently combining the fusion and fragmentation pictures.

The hadroproduction or resolved photoproduction of charmonium at finite $p_{T}$ via fusion proceeds at LO via the subprocesses $g g \rightarrow c \bar{c}+g, g q \rightarrow c \bar{c}+q$, and $q \bar{q} \rightarrow c \bar{c}+g$, where $q$ stands for a light quark or antiquark. The cross sections for the colour-singlet states $\left[\underline{1},{ }^{3} S_{1}\right]$ and $\left[\underline{1},{ }^{3} P_{J}\right]$ may be found in Ref. [3] and those for the colour-octet states $\left[\underline{8},{ }^{1} S_{0}\right],\left[\underline{8},{ }^{3} S_{1}\right]$, and $\left[\underline{8},{ }^{3} P_{J}\right]$ in Ref. [13]. As for direct photoproduction, the contributing subprocesses are $\gamma g \rightarrow c \bar{c}+g$ and $\gamma q \rightarrow c \bar{c}+q$, where the photon can either couple to the $q$ or $c$-quark line. The cross sections for the colour-singlet and most of the colour-octet states are presented in Refs. [23] and [11], respectively. The cross section of $\gamma g \rightarrow c \bar{c}\left[\underline{8},{ }^{3} S_{1}\right]+g$, which is not explicitly listed in Ref. [11], is obtained from the one of $\gamma g \rightarrow c \bar{c}\left[\underline{1},{ }^{3} S_{1}\right]+g$ through multiplication with $15 / 8$. At LO, $\left[\underline{1},{ }^{3} S_{1}\right]$ can only be produced through $g g \rightarrow$ $c \bar{c}+g$ and $\gamma g \rightarrow c \bar{c}+g$. Furthermore, $\left[\underline{1},{ }^{3} P_{J}\right]$ cannot be generated via $\gamma g \rightarrow c \bar{c}+g$ and $\gamma q \rightarrow c \bar{c}+q$, i.e. the direct photoproduction of $\chi_{c J}$ mesons is a pure colour-octet process.

The direct and resolved photoproduction of $J / \psi$ and $\chi_{c J}$ mesons via fragmentation was extensively discussed in Refs. 224,25,26]. The analysis of resolved photoproduction 25, 26] may be readily converted to hadroproduction by replacing the photon PDF's with the respective proton PDF's. The relevant partonic cross sections are available at nextto-leading order (NLO) in massless QCD [27]. The $d_{i \rightarrow n}$ functions in Eq. (2) pertinent to $i=g, c, \bar{c}$ and $n=\left[\underline{1},{ }^{3} S_{1}\right],\left[\underline{1},{ }^{3} P_{J}\right],\left[\underline{8},{ }^{3} S_{1}\right]$ were found in Ref. [28] and collected in the Appendix of Ref. [25]. Since the gluon is a $\left[\underline{8},{ }^{3} S_{1}\right]$ state, $\left.d_{g \rightarrow[8},{ }^{3} S_{1}\right]$ is of $\mathcal{O}\left(\alpha_{s}\right)$, while $d_{g \rightarrow\left[\underline{8},{ }^{1} S_{0}\right]}$ and $d_{g \rightarrow\left[\underline{8},{ }^{3} P_{J}\right]}$ require the emission of at least one extra gluon and are thus of $\mathcal{O}\left(\alpha_{s}^{2}\right)$ and beyond. As $\left\langle\mathcal{O}^{J / \psi}\left[\underline{8},{ }^{1} S_{0}\right]\right\rangle,\left\langle\mathcal{O}^{J / \psi}\left[\underline{8},{ }^{3} S_{1}\right]\right\rangle$, and $\left\langle\mathcal{O}^{J / \psi}\left[\underline{8},{ }^{3} P_{J}\right]\right\rangle$ all scale as $v^{7}$, it hence follows that the fragmentation process $g \rightarrow c \bar{c}\left[\underline{8},{ }^{3} S_{1}\right]$ dominates, while the other gluon-initiated colour-octet contributions are marginal. The $d_{i \rightarrow n}$ functions for $i=c, \bar{c}$ are of $\mathcal{O}\left(\alpha_{s}^{2}\right)$, so that the colour-octet contributions due to charm fragmentation are suppressed by $v^{4}$ relative to the corresponding colour-singlet contributions. In contrast to the fusion picture, it is therefore justified to disregard the $\left[\underline{8},{ }^{1} S_{0}\right]$ and $\left[\underline{8},{ }^{3} P_{J}\right]$ states in the fragmentation picture, as was done in Refs. [24,25,26].

We take the renormalization scale $\mu$ and the common factorization scale $M_{f}$ to be $\mu=M_{f}=m_{T}$, where $m_{T}$ is defined above Eq. (2). We define the starting scale $\mu_{0}$ of the FF's as $\mu_{0}=2 m_{c}=M_{J / \psi}$. For our LO analysis, we choose CTEQ4L [29] and GRV-LO [30] as the proton and photon PDF's, respectively, and evaluate $\alpha_{s}$ from the one-loop formula with $\Lambda^{(4)}=236 \mathrm{MeV}$ [29]. Whenever we work at NLO, we adopt the $\overline{\mathrm{MS}}$ renormalization and factorization scheme and employ CTEQ4M [29], GRV-HO [30], and the two-loop formula for $\alpha_{s}$ with $\Lambda \frac{(4)}{\mathrm{MS}}=296 \mathrm{MeV}$ [29]. In the case of fragmentation, 
we start from the NLO partonic cross sections calculated in the $\overline{\mathrm{MS}}$ scheme with $n_{f}=4$ massless flavours [27, and modify the factorization scheme for the collinear singularities associated with final-state charm quarks so as to match the finite- $m_{c}$ calculation. This procedure, which was proposed in Ref. [31], is equivalent to the matching between the massless-charm calculation in connection with the perturbative FF's of Ref. [32] and the massive-charm calculation without FF's.

Unfortunately, not all ingredients which would be necessary for a fully consistent NLO analysis are yet available. In the case of fusion, the NLO corrections to the cross sections $(d \sigma / d t)(i j \rightarrow c \bar{c}+k)$ in Eq. (1) are only known for direct photoproduction in the CSM [33]. Furthermore, in the case of fragmentation, the NLO corrections to the $d_{i \rightarrow n}$ functions in Eq. (2)) are still unknown.

In Ref. [33], it was found that, under typical HERA conditions, the bulk of the NLO corrections to the inclusive cross section of direct $J / \psi$ photoproduction in the CSM is due to the shift in $\left\langle\mathcal{O}^{J / \psi}\left[\underline{1},{ }^{3} S_{1}\right]\right\rangle$ which occurs if the QCD correction $(K)$ factor 18 is included in Eq. (3), while the genuine NLO corrections arising from the partonic cross sections in Eq. (1) and the proton PDF's only amount to about $20 \%$ in the inelastic regime $z \lesssim 0.9$. Here, $z$ is the inelasticity variable defined as $z=p_{p} \cdot p_{J / \psi} / p_{p} \cdot p_{\gamma}$, where $p_{p}, p_{\gamma}$, and $p_{J / \psi}$ are the proton, photon, and $J / \psi$ four-momenta, respectively. In our recent study of inclusive $J / \psi$ photoproduction via fragmentation [25], where we included both the direct and resolved channels, we found $K$ factors of about 0.9 and 1.8 for the colour-singlet and colour-octet processes, respectively. Guided by the observations made in Ref. [33], we do not expect the genuine NLO corrections to the $d_{i \rightarrow n}$ functions in Eq. (2), which are still missing, to drastically change these findings. Vice versa, we infer from the fragmentation results of Ref. [25] that, in the case of fusion, the unknown $K$ factors for direct photoproduction via the colour-octet mechanism and for resolved photoproduction via the colour-singlet and colour-octet mechanisms should also be of perturbative magnitude. In the case of direct photoproduction via the colour-octet mechanism, this expectation is also supported by a recent NLO calculation of the total cross section 34. In fact, at the typical photon-proton $\mathrm{CM}$ energy $W=100 \mathrm{GeV}$, the $K$ factors in the $\left[\underline{8},{ }^{1} S_{0}\right]$, $\left[\underline{8},{ }^{3} P_{0}\right]$, and $\left[\underline{8},{ }^{3} P_{2}\right]$ channels turned out to be as low as $1.26,1.05$, and 1.31 , respectively [34. We conclude that, for inclusive charmonium photoproduction at HERA, the present uncertainty in the size of the colour-octet matrix elements is likely to be more significant than the partial lack of knowledge of the genuine NLO corrections to the partonic cross sections in Eq. (11) and the $d_{i \rightarrow n}$ functions in Eq. (2).

The situation should be very different for inclusive charmonium hadroproduction at the Tevatron, especially in the low- $p_{T}$ range, where one expects substantial HO QCD effects due to multiple-gluon radiation. Such effects were estimated for the fusion mechanism in Ref. [16] by means of the Monte Carlo event generator PYTHIA [35] after implementing therein the relevant colour-octet processes, and they were indeed found to be very sizeable. The resulting $K$ factor for the $p_{T}$ distribution of prompt $J / \psi$ mesons may be extracted 
from Fig. 1 of Ref. [16] and is conveniently parameterized as

$$
K\left(p_{T}\right)=2.88-2.20 \cdot\left(\frac{p_{T}}{10 \mathrm{GeV}}-1\right)+1.04 \cdot\left(\frac{p_{T}}{10 \mathrm{GeV}}-1\right)^{2} .
$$

It ranges between 4 and 2 for $5 \mathrm{GeV}<p_{T}<15 \mathrm{GeV}$. We assume that this $K$ factor also approximately applies to the $\left[\underline{1},{ }^{3} S_{1}\right],\left[\underline{8},{ }^{1} S_{0}\right],\left[\underline{8},{ }^{3} S_{1}\right]$, and $\left[\underline{8},{ }^{3} P_{J}\right]$ channels separately. Thus, in order to estimate the HO-improved cross section of inclusive $J / \psi$ hadroproduction via fusion, we consistently evaluate these channels at LO and in turn multiply them by the $K$ factor of Eq. (5). On the other hand, our approximate NLO treatment of hadroproduction via fragmentation emerges from the corresponding analysis of resolved photoproduction [25.26] by replacing the photon PDF's with proton PDF's. If we extrapolate Eq. (5) to $p_{T}=20 \mathrm{GeV}$, we obtain $K=1.72$. This result is comparable to the value 1.45 , which we find for the dominant fragmentation channel, $\left[\underline{8},{ }^{3} S_{1}\right]$, using the same value for $\left\langle\mathcal{O}^{J / \psi}\left[\underline{8},{ }^{3} S_{1}\right]\right\rangle$ both at LO and NLO. This indicates that the duality of the fusion and fragmentation pictures also carries over to higher orders.

\section{Fit to the data of charmonium hadroproduction}

We now describe our fitting procedure. As experimental input, we use the latest CDF data samples of prompt $J / \psi$ mesons and of $J / \psi$ mesons originating from the radiative decays $\chi_{c J} \rightarrow J / \psi+\gamma$ of prompt $\chi_{c J}$ mesons [2]. The $J / \psi$ mesons were detected via their $J / \psi \rightarrow \mu^{+} \mu^{-}$decays. The data were collected in $p \bar{p}$ collisions with centre-of-mass $(\mathrm{CM})$ energy $\sqrt{s}=1.8 \mathrm{TeV}$ at the Fermilab Tevatron and come as the differential cross section $d \sigma / d p_{T}$ integrated over the rapidity range $|\eta|<0.6$. Each data sample consists of 11 data points ranging from $p_{T}=5.24 \mathrm{GeV}$ to $18.38 \mathrm{GeV}$. We adopt the measured values of the branching fractions $B\left(\chi_{c J} \rightarrow J / \psi+\gamma\right)$ and $B\left(J / \psi \rightarrow \mu^{+} \mu^{-}\right)$from Ref. [36]. We determine $\left\langle\mathcal{O}^{J / \psi}\left[\underline{1},{ }^{3} S_{1}\right]\right\rangle$ from the experimental value of $\Gamma\left(J / \psi \rightarrow \ell^{+} \ell^{-}\right)$[36] via Eq. (3). Our LO and NLO results are listed in Table 1.

We first concentrate on prompt $J / \psi$ hadroproduction. In Refs. [13,21], the $J / \psi$ colouroctet matrix elements were fitted on the basis of the fusion picture at LO, whereby the $\left[\underline{8},{ }^{3} S_{1}\right]$ component was supplemented with leading logarithms from $g \rightarrow c \bar{c}\left[\underline{8},{ }^{3} S_{1}\right]$ fragmentation. This was achieved in an ad-hoc way, namely by multiplication with the ratio of the corresponding gluon-fragmentation cross sections with and without LO AP evolution. Here, we adopt a more rigorous approach. We introduce a variable separation cut $p_{T}^{\text {cut }}$, which we choose to coincide with any of the $11 \mathrm{CDF}$ data points, as a demarcation between the fusion and fragmentation pictures. In the first step, we determine $\left\langle\mathcal{O}^{J / \psi}\left[\underline{8},{ }^{3} S_{1}\right]\right\rangle$ by fitting the data points for $p_{T} \geq p_{T}^{\text {cut }}$ in the fragmentation picture. This result is then used as input for the second step, in which $\left\langle\mathcal{O}^{J / \psi}\left[\underline{8},{ }^{1} S_{0}\right]\right\rangle$ and $\left\langle\mathcal{O}^{J / \psi}\left[\underline{8},{ }^{3} P_{0}\right]\right\rangle$ are determined by fitting the data points for $p_{T} \leq p_{T}^{\text {cut }}$ in the fusion picture. As was noticed in Ref. [13], the cross sections of $c \bar{c}\left[\underline{8},{ }^{1} S_{0}\right]$ and $c \bar{c}\left[\underline{8},{ }^{3} P_{0}\right]$ production have very similar $p_{T}$ dependences, so that treating $\left\langle\mathcal{O}^{J / \psi}\left[\underline{8},{ }^{1} S_{0}\right]\right\rangle$ and $\left\langle\mathcal{O}^{J / \psi}\left[\underline{8},{ }^{3} P_{0}\right]\right\rangle$ as independent fit parameters would yield highly correlated results. It was therefore suggested [13] to fix a specific linear 
Table 1: Values of the $J / \psi$ matrix elements and of $r$ resulting from the LO and HOimproved fits to the CDF data [2]. $M_{r}^{J / \psi}$ is defined in Eq. (6). The first 10 (last 2) data points are described in the fusion (fragmentation) picture.

\begin{tabular}{|c|c|c|c|}
\hline \hline & LO & HO & scaling \\
\hline$\left\langle\mathcal{O}^{J / \psi}\left[\underline{1},{ }^{3} S_{1}\right]\right\rangle$ & $(7.63 \pm 0.54) \cdot 10^{-1} \mathrm{GeV}^{3}$ & $(1.30 \pm 0.09) \mathrm{GeV}^{3}$ & {$\left[m_{c}^{3} v^{3}\right]$} \\
$\left\langle\mathcal{O}^{J / \psi}\left[\underline{8},{ }^{3} S_{1}\right]\right\rangle$ & $(3.94 \pm 0.63) \cdot 10^{-3} \mathrm{GeV}^{3}$ & $(2.73 \pm 0.45) \cdot 10^{-3} \mathrm{GeV}^{3}$ & {$\left[m_{c}^{3} v^{7}\right]$} \\
$M_{r}^{J / \psi}$ & $(6.52 \pm 0.67) \cdot 10^{-2} \mathrm{GeV}^{3}$ & $(5.72 \pm 1.84) \cdot 10^{-3} \mathrm{GeV}^{3}$ & {$\left[m_{c}^{3} v^{7}\right]$} \\
$r$ & 3.47 & 3.54 & \\
$\chi_{\mathrm{DF}}^{2}$ fus. & $5.97 / 10$ & $2.23 / 10$ & \\
$\chi_{\mathrm{DF}}^{2}$ fra. & $1.53 / 2$ & $1.73 / 2$ & \\
$\chi_{\mathrm{DF}}^{2}$ tot. & $7.49 / 12$ & $3.96 / 12$ & \\
\hline \hline
\end{tabular}

combination of $\left\langle\mathcal{O}^{J / \psi}\left[\underline{8},{ }^{1} S_{0}\right]\right\rangle$ and $\left\langle\mathcal{O}^{J / \psi}\left[\underline{8},{ }^{3} P_{0}\right]\right\rangle$ instead. Following this suggestion, we define

$$
M_{r}^{J / \psi}=\left\langle\mathcal{O}^{J / \psi}\left[\underline{8},{ }^{1} S_{0}\right]\right\rangle+\frac{r}{m_{c}^{2}}\left\langle\mathcal{O}^{J / \psi}\left[\underline{8},{ }^{3} P_{0}\right]\right\rangle,
$$

where $r$ is to be chosen in such a way that the superposition of these two channels is insensitive to precisely how they are weighted relative to each other. We determine $r$ to be the ratio of the fit result for $\left\langle\mathcal{O}^{J / \psi}\left[\underline{8},{ }^{1} S_{0}\right]\right\rangle$ under the condition $\left\langle\mathcal{O}^{J / \psi}\left[\underline{8},{ }^{3} P_{0}\right]\right\rangle=0$ to the one for $\left\langle\mathcal{O}^{J / \psi}\left[\underline{8},{ }^{3} P_{0}\right]\right\rangle / m_{c}^{2}$ under the condition $\left\langle\mathcal{O}^{J / \psi}\left[\underline{8},{ }^{1} S_{0}\right]\right\rangle=0$. We fit by analytically minimizing the $\chi^{2}$ values. We measure the overall quality of the fit by adding the two $\chi^{2}$ values achieved in the determination of $\left\langle\mathcal{O}^{J / \psi}\left[\underline{8},{ }^{3} S_{1}\right]\right\rangle$ in the upper $p_{T}$ range and of $M_{r}^{J / \psi}$ in the lower $p_{T}$ range. Finally, we determine $p_{T}^{\text {cut }}$ by minimizing the total $\chi^{2}$. It turns out that the best results are obtained if the first 10 (last 2) data points are described according to the fusion (fragmentation) picture, i.e. the transition between these two pictures happens somewhere around $p_{T}=15 \mathrm{GeV}$.

Figures 1(a) and (b) illustrate for the LO and HO-improved analyses, respectively, how the fusion and fragmentation cross section compare with the experimental data and how they are decomposed into their colour-singlet and colour-octet components. The fragmentation results are only shown for $p_{T} \geq 10.91 \mathrm{GeV}$, while the fusion results are displayed over the full $p_{T}$ range. In each case, the total result (solid lines) is obtained as the superposition of the colour-singlet (dotted lines) and colour-octet contributions. The $\left[\underline{8},{ }^{3} S_{1}\right]$ contributions (dashed lines) of the fusion and fragmentation pictures almost coincide. The colour-octet contribution proportional to $M_{r}^{J / \psi}$ (dot-dashed line) is only included in the fusion picture, while it is neglected in the fragmentation picture for reasons explained in Section 2. Notice that the LO $\left[\underline{1},{ }^{3} S_{1}\right]$ contributions in the fusion and fragmentation pictures arise from very different sources. On the one hand, the LO fusion subprocess $g g \rightarrow c \bar{c}+g$ has no counterpart in the fragmentation picture. On the other hand, the LO fragmentation processes $g \rightarrow c \bar{c}+g g, c \rightarrow c \bar{c}+c$, and $\bar{c} \rightarrow c \bar{c}+\bar{c}$ 
correspond to $\mathrm{HO}$ contributions in the fusion picture. This explains why the dotted lines in Figs. 1 (a) and (b) do not match. As is by now common knowledge, the colour-octet processes are indeed necessary in order to reconcile theory with experiment as far as inclusive hadroproduction of prompt charmonium at the Tevatron is concerned. The results and the various $\chi^{2}$ values of the LO and HO-improved fits are summarized in Table 1 . The errors quoted in Table 1 only include the experimental errors on the CDF data. In addition, there are the usual theoretical uncertainties due to the dependence on the renormalization and factorization scales and on the proton PDF's, which have been carefully estimated in Ref. [21]. Our LO results should be compared with those obtained in Refs. [13,21]. We find reasonable agreement for $M_{r}^{J / \psi}$ (and $r$ ), which was determined to be $M_{3}^{J / \psi}=(6.6 \pm 1.5) \cdot 10^{-2} \mathrm{GeV}^{3}$ in Ref. [13] and $M_{3.5}^{J / \psi}=(4.38 \pm 1.15) \cdot 10^{-2} \mathrm{GeV}^{3}$ (for CTEQ4L [29]) in Ref. [21]. On the other hand, our result for $\left\langle\mathcal{O}^{J / \psi}\left[\underline{8},{ }^{3} S_{1}\right]\right\rangle$ is somewhat smaller than the value $(6.6 \pm 2.1) \cdot 10^{-3} \mathrm{GeV}^{3}$ of Ref. 13 and considerably smaller than the value $(1.06 \pm 0.14) \cdot 10^{-2} \mathrm{GeV}^{3}$ of Ref. [21]. We attribute this deviation to the different implementations of the fragmentation contribution. As we pass from LO to our approximate $\mathrm{HO}$ implementation, we observe that $\left\langle\mathcal{O}^{J / \psi}\left[\underline{8},{ }^{3} S_{1}\right]\right\rangle$ is only slightly decreased, by $31 \%$, while $M_{r}^{J / \psi}$ drops off by more than one order of magnitude. The change in $\left\langle\mathcal{O}^{J / \psi}\left[\underline{8},{ }^{3} S_{1}\right]\right\rangle$ precisely compensates the enhancement due to the $K$ factor in the $g \rightarrow c \bar{c}\left[\underline{8},{ }^{3} S_{1}\right]$ fragmentation channel at high $p_{T}$. The dramatic reduction of $M_{r}^{J / \psi}$ is due to the interplay of two effects. On the one hand, the $\left[\underline{8},{ }^{3} S_{1}\right]$ fusion channel, whose matrix element is fixed by the fit in the fragmentation regime, is strongly enhanced at low $p_{T}$ due to multiple-gluon radiation according to Eq. (5) and only leaves little room for possible contributions due the $\left[\underline{8},{ }^{1} S_{0}\right]$ and $\left[\underline{8},{ }^{3} P_{J}\right]$ fusion channels. On the other hand, the latter channels are also strongly enhanced at low $p_{T}$ by the $K$ factor of Eq. (5), so that $M_{r}^{J / \psi}$ is even further reduced. The situation is nicely illustrated if we compare the relative importance of the various colour-octet fusion contributions in Figs. 1(a) and (b). At LO, the combined $\left[\underline{8},{ }^{1} S_{0}\right]$ and $\left[\underline{8},{ }^{3} P_{J}\right]$ contributions exceed the one due to the $\left[\underline{8},{ }^{3} S_{1}\right]$ channel way up to $p_{T}=10 \mathrm{GeV}$, while, at $\mathrm{HO}$, the $\left[\underline{8},{ }^{3} S_{1}\right]$ contribution is dominant over the full $p_{T}$ range considered. Our HO-improved results for $\left\langle\mathcal{O}^{J / \psi}\left[\underline{8},{ }^{3} S_{1}\right]\right\rangle$ and $M_{r}^{J / \psi}$ are very similar to those extracted in Ref. [16] from the previous CDF data [1] with the 1994 GRV-HO proton PDF's [37], which correctly account for the low- $x$ behaviour of the proton structure function $F_{2}\left(x, Q^{2}\right)$ measured at HERA, namely $\left\langle\mathcal{O}^{J / \psi}\left[\underline{8},{ }^{3} S_{1}\right]\right\rangle=(3.4 \pm 0.4) \cdot 10^{-3} \mathrm{GeV}^{3}$ and $M_{3}^{J / \psi}=(6.0 \pm 1.2) \cdot 10^{-3} \mathrm{GeV}^{3}$. Finally, we note that the overall $\chi^{2}$ per degree of freedom $\left(\chi_{\mathrm{DF}}^{2}\right)$ is significantly reduced, from 0.62 to 0.33 , as we pass from LO to HO.

In the remainder of this section, we discuss the extraction of the $\chi_{c J}$ matrix elements through a fit to the CDF data on prompt $\chi_{c J}$ mesons [2]. For simplicity, we work at LO in the fusion picture. The fit results for $\left\langle\mathcal{O}^{\chi_{c 0}}\left[\underline{1},{ }^{3} P_{0}\right]\right\rangle$ and $\left\langle\mathcal{O}^{\chi_{c 0}}\left[\underline{8},{ }^{3} S_{1}\right]\right\rangle$ are displayed in Table 2. The quality of the fit is rather high, with $\chi_{\mathrm{DF}}^{2}=0.35$. Our value for $\left\langle\mathcal{O}^{\chi_{c 0}}\left[\underline{1},{ }^{3} P_{0}\right]\right\rangle$ is somewhat larger than those extracted from the partial widths of the $\chi_{c J}$-meson decays to light hadrons [19] and two photons [20]. In turn, our value for $\left\langle\mathcal{O}^{\chi_{c 0}}\left[\underline{8},{ }^{3} S_{1}\right]\right\rangle$ is smaller than the one found in Ref. [13]. The interplay of the colour-singlet and color-octet processes 
Table 2: Values of the $\chi_{c J}$ matrix elements resulting from the LO fit to the CDF data [2]. All 11 data points are described in the fusion picture.

\begin{tabular}{|c|c|c|}
\hline \hline & LO & scaling \\
\hline$\left\langle\mathcal{O}^{\chi_{c 0}}\left[\underline{1},{ }^{3} P_{0}\right]\right\rangle$ & $(2.29 \pm 0.25) \cdot 10^{-1} \mathrm{GeV}^{5}$ & {$\left[m_{c}^{5} v^{5}\right]$} \\
$\left\langle\mathcal{O}^{\chi_{c 0}}\left[\underline{8},{ }^{3} S_{1}\right]\right\rangle$ & $(6.81 \pm 1.75) \cdot 10^{-4} \mathrm{GeV}^{3}$ & {$\left[m_{c}^{3} v^{5}\right]$} \\
$\chi_{\text {DF }}^{2}$ fus. & $3.82 / 11$ & \\
\hline \hline
\end{tabular}

and the comparison of their superposition with the CDF data is illustrated in Fig. 2.

\section{Predictions for charmonium photoproduction}

Having extracted the leading $J / \psi$ and $\chi_{c J}$ colour-octet matrix elements from the latest CDF data taking into account information on HO QCD effects, we are now in a position to explore the phenomenological consequences for inclusive charmonium photoproduction in $e p$ collisions at HERA. HERA is presently operated in such a way that $E_{e}=27.5 \mathrm{GeV}$ positrons collide with $E_{p}=820 \mathrm{GeV}$ protons in the laboratory frame, so that approximately $\sqrt{s}=300 \mathrm{GeV}$ is available in the $\mathrm{CM}$ system. In the case of photoproduction, the positrons act as a source of energetic, quasi-real photons, whose energy distribution is well described in the Weizsäcker-Williams approximation [38] by a well-known formula, which may be found e.g. in Eq. (17) of Ref. [25].

The H1 [14] and ZEUS [15] collaborations recently presented their measurements of inelastic $J / \psi$ photoproduction. In both experiments, the scattered positron was not tagged, so that the maximum photon virtuality was $Q_{\max }^{2}=4 \mathrm{GeV}^{2}$. The measured $e p$ cross sections were converted to averaged $\gamma p$ cross sections by dividing out the photonflux factor, which was evaluated by integrating the Weizsäcker-Williams formula over the considered photon-energy range. The experimental information was presented in the form of distributions differential in the photon-proton CM energy $W$, the $J / \psi$ transverse momentum $p_{T}$, and the inelasticity variable $z$. We adhere to the kinematic cuts used in the ZEUS analysis, namely $0.4<z<0.8$ and $p_{T}^{2}>1 \mathrm{GeV}^{2}$ for the $W$ distribution, $0.4<z<0.9$ and $50 \mathrm{GeV}<W<180 \mathrm{GeV}$ for the $p_{T}^{2}$ distribution, and $p_{T}^{2}>1 \mathrm{GeV}^{2}$ and $50 \mathrm{GeV}<W<180 \mathrm{GeV}$ for the $z$ distribution. The photon-flux factor corresponding to this $W$ interval is 0.106. By contrast, the $\mathrm{H} 1$ data refer the $W$ interval $30 \mathrm{GeV}<W<$ $150 \mathrm{GeV}$, for which the flux factor is 0.150 . Actually, the ZEUS measurement only reached down to $z>0.4$ and was extrapolated to $z=0$ by Monte Carlo simulations yielding an enhancement factor of 1.10 [15]. The H1 data were treated in a similar way. We undo this artificial extrapolation and divide the published $W$ and $p_{T}^{2}$ distributions [14, 15] by this factor. The contribution due to $\psi^{\prime}$ mesons with subsequent decay into $J / \psi$ mesons was not subtracted from the ZEUS data. As in Ref. [15], we thus multiply the theoretical predictions by an overall factor of 1.15 to approximately account for this contribution. 
The data are mostly concentrated in the low- $p_{T}$ range, where the fusion picture should be valid.

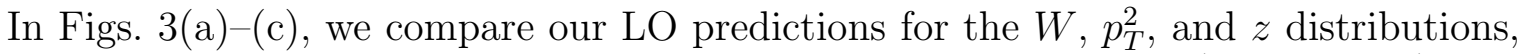
respectively, with the ZEUS and H1 data. The circumstance that $\left\langle\mathcal{O}^{J / \psi}\left[\underline{8},{ }^{1} S_{0}\right]\right\rangle$ and $\left\langle\mathcal{O}^{J / \psi}\left[\underline{8},{ }^{3} P_{0}\right]\right\rangle$ are not separately fixed by the fit to the CDF data induces some uncertainty in the colour-octet contributions to the cross sections of direct and resolved photoproduction and thus also in the total cross section. This uncertainty is encompassed by the results for $\left\langle\mathcal{O}^{J / \psi}\left[\underline{8},{ }^{1} S_{0}\right]\right\rangle=M_{r}^{J / \psi}$ and $\left\langle\mathcal{O}^{J / \psi}\left[\underline{8},{ }^{3} P_{0}\right]\right\rangle=0$ and those for $\left\langle\mathcal{O}^{J / \psi}\left[\underline{8},{ }^{1} S_{0}\right]\right\rangle=0$ and $\left\langle\mathcal{O}^{J / \psi}\left[\underline{8},{ }^{3} P_{0}\right]\right\rangle=\left(m_{c}^{2} / r\right) M_{r}^{J / \psi}$, which are actually shown in Figs. 苟(a)-(c). From Fig. 通(c), we observe that the colour-octet contribution of direct photoproduction is dominant for $z \gtrsim 0.5$. Thus, it also makes up the bulk of the cross sections shown in Fig. 3(a) and (b), which are integrated over $0.4<z<0.8$ and $0.4<z<0.9$, respectively. As is evident from Figs. B(b) and (c), this contribution is also responsible for the significant excess of the LO predictions over the experimental results for $d \sigma / d p_{T}^{2}$ and $d \sigma / d z$ at low $p_{T}$ and high $z$, respectively. (By contrast, such an excess does not show up in Fig. 3(a), where the $z$ integration only extends up to 0.8.) This observation was assessed in the ZEUS publication 15] by the statement that the predictions of a specific leading-order colour-octet model, as formulated to describe the CDF data on $J / \psi$ hadroproduction, are not consistent with the data. To our mind, the salient point is that those predictions were made on the basis of LO fits to the CDF data. It is crucial to notice that the colour-octet contribution of direct photoproduction is essentially due the $\left[\underline{8},{ }^{1} S_{0}\right],\left[\underline{8},{ }^{3} P_{0}\right]$, and $\left[\underline{8},{ }^{3} P_{2}\right]$ channels [10, 11] and thus proportional to $M_{r}^{J / \psi}$. In Section 3, we have seen that the fit result for $M_{r}^{J / \psi}$ is greatly reduced by the inclusion of HO QCD effects on hadroproduction at low $p_{T}$ [16]. This nourishes the hope that the apparent discrepancy between theory and experiment at HERA may thus be alleviated at higher orders.

In Figs. $\frac{6}{4}(\mathrm{a})-(\mathrm{c})$, we take a first step towards a full NLO description of the HERA data on inelastic $J / \psi$ photoproduction by repeating the analyses of Figs. $3($ a) - (c) using the HO-improved results of Table 1, the NLO proton and photon PDF's specified in Section 2, and the two-loop formula for $\alpha_{s}$. However, we do not include the NLO corrections to the partonic cross sections in Eq. (11) As already mentioned in Section 2, they are unknown, except for direct photoproduction in the CSM [33]. In the latter case, they were found to moderately increase the cross section, by about $20 \%$ [33. We observe that the HOimproved predictions tend to undershoot the data leaving room for a substantial $K$ factor due to the missing NLO corrections to the partonic cross sections in Eq. (1). Now, the colour-singlet contribution of direct photoproduction, which is well under theoretical control [33], is by far dominant, except in the corners of phase space, at $z \lesssim 0.15$ and $z \gtrsim 0.85$, where the colour-octet contributions of resolved and direct photoproduction, respectively, take over. It has been noticed [25,26, 39] that special care must be exercised in the treatment of the latter contributions. In the vicinity of the upper endpoint, $\mathrm{HO}$ nonperturbative contributions are enhanced and lead to a breakdown of the NRQCD expansion, so that only an average cross section over a sufficiently large region close to $z=1$ can be predicted [39]. On the other hand, one my infer from the study of resolved 
$J / \psi$ photoproduction via fragmentation [25,26] that the corresponding partonic cross sections in Eq. (1) are likely to receive significant NLO corrections at low $z$. Of course, we should also bear in mind that the predictions shown in Figs. 3 and 1 still suffer from considerable theoretical uncertainties related to the choice of the scales $\mu$ and $M_{f}$, the PDF's, and other input parameters such as $m_{c}$ and $\Lambda^{(4)}$ [21.33]. From these observations, we conclude that it is premature at this point to speak about a discrepancy between the Tevatron [1,2] and HERA [14,15] data of inclusive $J / \psi$ production within the framework of NRQCD [4].

Finally, we present LO predictions for the inclusive photoproduction of $\chi_{c J}$ mesons with subsequent radiative feed down to $J / \psi$ mesons. We work at LO in the fusion picture using the $\chi_{c J}$ matrix elements of Table 2. In Figs. 5(a)-(c), we present our results for the $W, p_{T}^{2}$, and $z$ distributions, respectively, under the same kinematical conditions as in the $J / \psi$ case considered in Figs. 3 and 4 . Notice that direct photoproduction of $\chi_{c J}$ mesons is prohibited at LO in the CSM. From Fig. 5(c), we observe that, except for $z \gtrsim 0.8$, the dominant contribution arises from resolved photoproduction in the CSM. Comparing Figs. 5(a) and (b) with Figs. $3(\mathrm{a}$ ) and (b), we see that, after integration over $z$, this indirect source of $J / \psi$ mesons is suppressed by roughly two orders of magnitude relative to prompt $J / \psi$ production.

\section{Conclusions}

We determined the $J / \psi$ colour-octet matrix elements which appear in the NRQCD expansion [4] at leading order in $v$ by fitting the latest Tevatron data of prompt $J / \psi$ hadroproduction [2]. We found that the result for the linear combination $M_{r}^{J / \psi}$ of $\left\langle\mathcal{O}^{J / \psi}\left[\underline{8},{ }^{1} S_{0}\right]\right\rangle$ and $\left\langle\mathcal{O}^{J / \psi}\left[\underline{8},{ }^{3} P_{0}\right]\right\rangle$ is substantially reduced if the HO QCD effects due to the multiple emission of gluons, which had been estimated by Monte Carlo techniques [16], are taken into account. As an important consequence, the intriguing excess of the LO NRQCD prediction for inelastic $J / \psi$ photoproduction at $z$ close to unity [10,11] over the HERA measurements [14,15] disappears. We assess this finding as an indication that it is premature to proclaim an experimental falsification of the NRQCD framework on the basis of the HERA data. Although we believe that our analysis captures the main trend of the $\mathrm{HO}$ improvement, we stress that it is still at an exploratory level, since a number of ingredients which would be necessary for a fully consistent NLO treatment of inclusive $J / \psi$ hadroproduction and photoproduction are still missing.

\section{ACKNOWLEDGMENTS}

One of us (G.K.) is grateful to the Theory Group of the Werner-Heisenberg-Institut for the hospitality extended to him during a visit when this paper was prepared.

\section{References}


[1] CDF Collaboration, F. Abe et al., Phys. Rev. Lett. 69 (1992) 3704; 71 (1993) 2537; 75 (1995) 1451.

[2] CDF Collaboration, F. Abe et al., Phys. Rev. Lett. 79 (1997) 578.

[3] R. Baier and R. Rückl, Phys. Lett. 102 B (1980) 364; Z. Phys. C 19 (1983) 251;

B. Humpert, Phys. Lett. B 184 (1987) 105;

R. Gastmans, W. Troost and T.T. Wu, Phys. Lett. B 184 (1987) 257; Nucl. Phys. B 291 (1987) 731.

[4] G.T. Bodwin, E. Braaten and G.P. Lepage, Phys. Rev. D 51 (1995) 1125; 55 (1997) $5855(\mathrm{E})$.

[5] E. Braaten and Y.-Q. Chen, Phys. Rev. Lett. 76 (1996) 730;

F. Yuan, C.-F. Qiao and K.-T. Chao, Phys. Rev. D 56 (1997) 1663.

[6] K. Cheung, W.-Y. Keung and T.C. Yuan, Phys. Rev. Lett. 76 (1996) 877;

P. Cho, Phys. Lett. B 368 (1996) 171;

C.-F. Qiao, F. Yuan and K.-T. Chao, Phys. Rev. D 55 (1997) 4001.

[7] W.-K. Tang and M. Vänttinen, Phys. Rev. D 53 (1996) 4851; 54 (1996) 4349;

M. Beneke and I.Z. Rothstein, Phys. Rev. D 54 (1996) 2005;

S. Fleming and I. Maksymyk, Phys. Rev. D 54 (1996) 3608;

S. Gupta and K. Sridhar, Phys. Rev. D 54 (1996) 5545.

[8] P. Ko, J. Lee and H.S. Song, Phys. Rev. D 53 (1996) 1409;

S. Fleming, O.F. Hernández, I. Maksymyk and H. Nadeau, Phys. Rev. D 55 (1997) 4098.

[9] J. Amundson, S. Fleming and I. Maksymyk, Phys. Rev. D 56 (1997) 5844.

[10] M. Cacciari and M. Krämer, Phys. Rev. Lett. 76 (1996) 4128.

[11] P. Ko, J. Lee and H.S. Song, Phys. Rev. D 54 (1996) 4312;

J. Lee, private communication.

[12] DELPHI Collaboration, P. Abreu et al., Z. Phys. C 69 (1996) 575;

L3 Collaboration, O. Adriani et al., Phys. Lett. B 288 (1992) 412;

OPAL Collaboration, G. Alexander et al., Phys. Lett. B 384 (1996) 343.

[13] P. Cho and A.K. Leibovich, Phys. Rev. D 53 (1996) 150; 53 (1996) 6203.

[14] H1 Collaboration, S. Aid et al., Nucl. Phys. B 472 (1996) 3.

[15] ZEUS Collaboration, J. Breitweg et al., Z. Phys. C 76 (1997) 599;

R. Brugnera, private communication.

[16] B. Cano-Coloma and M.A. Sanchis-Lozano, Nucl. Phys. B 508 (1997) 753. 
[17] J. Binnewies, B.A. Kniehl and G. Kramer, Z. Phys. C 76 (1997) 677.

[18] R. Barbieri, R. Gatto, R. Kögerler and Z. Kunzst, Phys. Lett. 57 B (1975) 455; Nucl. Phys. B 105 (1976) 125.

[19] G.T. Bodwin, E. Braaten and G.P. Lepage, Phys. Rev. D 46 (1992) R1914;

M.L. Mangano and A. Petrelli, Phys. Lett. B 352 (1995) 445;

A. Petrelli, Phys. Lett. B 380 (1996) 159;

H.-W. Huang and K.-T. Chao, Phys. Rev. D 54 (1996) 6850; 56 (1997) 1821 (E); 55 (1997) 244.

[20] H.-W. Huang, C.-F. Qiao and K.-T. Chao, Phys. Rev. D 54 (1996) 2123.

[21] M. Beneke and M. Krämer, Phys. Rev. D 55 (1997) 5269.

[22] E. Braaten and S. Fleming, Phys. Rev. Lett. 74 (1995) 3327;

M. Cacciari, M. Greco, M.L. Mangano and A. Petrelli, Phys. Lett. B 356 (1995) 553.

[23] E.L. Berger and D. Jones, Phys. Rev. D 23 (1981) 1521.

[24] R.M. Godbole, D.P. Roy and K. Sridhar, Phys. Lett. B 373 (1996) 328.

[25] B.A. Kniehl and G. Kramer, Phys. Rev. D 56 (1997) 5820.

[26] B.A. Kniehl and G. Kramer, Phys. Lett. B 413 (1997) 416.

[27] P. Aurenche, R. Baier, A. Douiri, M. Fontannaz and D. Schiff, Nucl. Phys. B 286 (1987) 553;

F. Aversa, P. Chiappetta, M. Greco and J.Ph. Guillet, Nucl. Phys. B 327 (1989) 105.

[28] E. Braaten and T.C. Yuan, Phys. Rev. Lett. 71 (1993) 1673; Phys. Rev. D 50 (1994) $3176 ; 52$ (1995) 6627;

E. Braaten, K. Cheung and T.C. Yuan, Phys. Rev. D 48 (1993) 4230;

Y.-Q. Chen, Phys. Rev. D 48 (1993) 5181; 50 (1994) 6013 (E);

T.C. Yuan, Phys. Rev. D 50 (1994) 5664;

E. Braaten and Y.-Q. Chen, Phys. Rev. D 55 (1997) 2693.

[29] H.L. Lai, J. Huston, S. Kuhlmann, F. Olness, J. Owens, D. Soper, W.K. Tung and H. Weerts, Phys. Rev. D 55 (1997) 1280.

[30] M. Glück, E. Reya and A. Vogt, Phys. Rev. D 46 (1992) 1973.

[31] B.A. Kniehl, G. Kramer and M. Spira, Z. Phys. C 76 (1997) 689.

[32] B. Mele and P. Nason, Nucl. Phys. B 361 (1991) 626;

J.P. Ma, Nucl. Phys. B 506 (1997) 329.

[33] M. Krämer, J. Zunft, J. Steegborn and P.M. Zerwas, Phys. Lett. B 348 (1995) 657;

M. Krämer, Nucl. Phys. B 459 (1996) 3. 
[34] A. Petrelli, M. Cacciari, M. Greco, F. Maltoni and M.L. Mangano, Report No. CERN-TH/97-142, DESY 97-090 and hep-ph/9707223 (July 1997);

F. Maltoni, M.L. Mangano and A. Petrelli, Report No. CERN-TH/97-202 and hep ph/9708349 (August 1997).

[35] T. Sjöstrand, Comput. Phys. Commun. 82 (1994) 74.

[36] Particle Data Group, R.M. Barnett et al., Phys. Rev. D 54 (1996) 1.

[37] M. Glück, E. Reya and A. Vogt, Z. Phys. C 67 (1995) 433.

[38] E.J. Williams, Proc. Roy. Soc. London A 139 (1933) 163;

C.F. v. Weizsäcker, Z. Phys. 88 (1934) 612.

[39] M. Beneke, I.Z. Rothstein and M.B. Wise, Phys. Lett. B 408 (1997) 373. 


\section{FIGURE CAPTIONS}

Figure 1: (a) LO and (b) HO-improved fits to the CDF data on the inclusive hadroproduction of prompt $J / \psi$ mesons [2], which come in the form of $d \sigma / d p_{T}$ integrated over $|\eta|<0.6$ as a function of $p_{T}$. The fragmentation results are only shown for $p_{T} \geq 10.91 \mathrm{GeV}$, while the fusion results are displayed over the full $p_{T}$ range.

Figure 2: LO fit in the fusion picture to the CDF data on the inclusive hadroproduction of prompt $\chi_{c J}$ mesons with radiative feed down to $J / \psi$ mesons [2], which come in the form of $d \sigma / d p_{T}$ integrated over $|\eta|<0.6$ as a function of $p_{T}$.

Figure 3: The ZEUS data on the inclusive photoproduction of prompt $J / \psi$ mesons [15], which come in the form of (a) $\sigma$ integrated over $0.4<z<0.8$ and $p_{T}^{2}>1 \mathrm{GeV}^{2}$ as a function of $W$, (b) $d \sigma / d p_{T}^{2}$ integrated over $0.4<z<0.9$ and $50 \mathrm{GeV}<W<180 \mathrm{GeV}$ as a function of $p_{T}^{2}$, and (c) $d \sigma / d z$ integrated over $p_{T}^{2}>1 \mathrm{GeV}^{2}$ and $50 \mathrm{GeV}<W<$ $180 \mathrm{GeV}$ as a function of $z$, are compared with the respective LO predictions. The total results (solid lines) are built up by the direct-photon colour-singlet (dotted lines), directphoton colour-octet (dashed lines), resolved-photon colour-singlet (dot-dashed lines), and resolved-photon colour-octet (dot-dot-dashed lines) contributions. The respective $\mathrm{H} 1$ data [14], which refer to $30 \mathrm{GeV}<W<150 \mathrm{GeV}$, are also shown.

Figure 4: Same as in Fig. 3, but for the HO-improved predictions.

Figure 5: LO predictions for the inclusive photoproduction of prompt $\chi_{c J}$ mesons with radiative feed down to $J / \psi$ mesons in the form of (a) $\sigma$ integrated over $0.4<z<0.8$ and $p_{T}^{2}>1 \mathrm{GeV}^{2}$ as a function of $W$, (b) $d \sigma / d p_{T}^{2}$ integrated over $0.4<z<0.9$ and $50 \mathrm{GeV}<W<180 \mathrm{GeV}$ as a function of $p_{T}^{2}$, and (c) $d \sigma / d z$ integrated over $p_{T}^{2}>1 \mathrm{GeV}^{2}$ and $50 \mathrm{GeV}<W<180 \mathrm{GeV}$ as a function of $z$. The total results (solid lines) are built up by the direct-photon colour-octet (dashed lines), resolved-photon colour-singlet (dot-dashed lines), and resolved-photon colour-octet (dot-dot-dashed lines) contributions. Direct photoproduction is prohibited at LO in the CSM. 


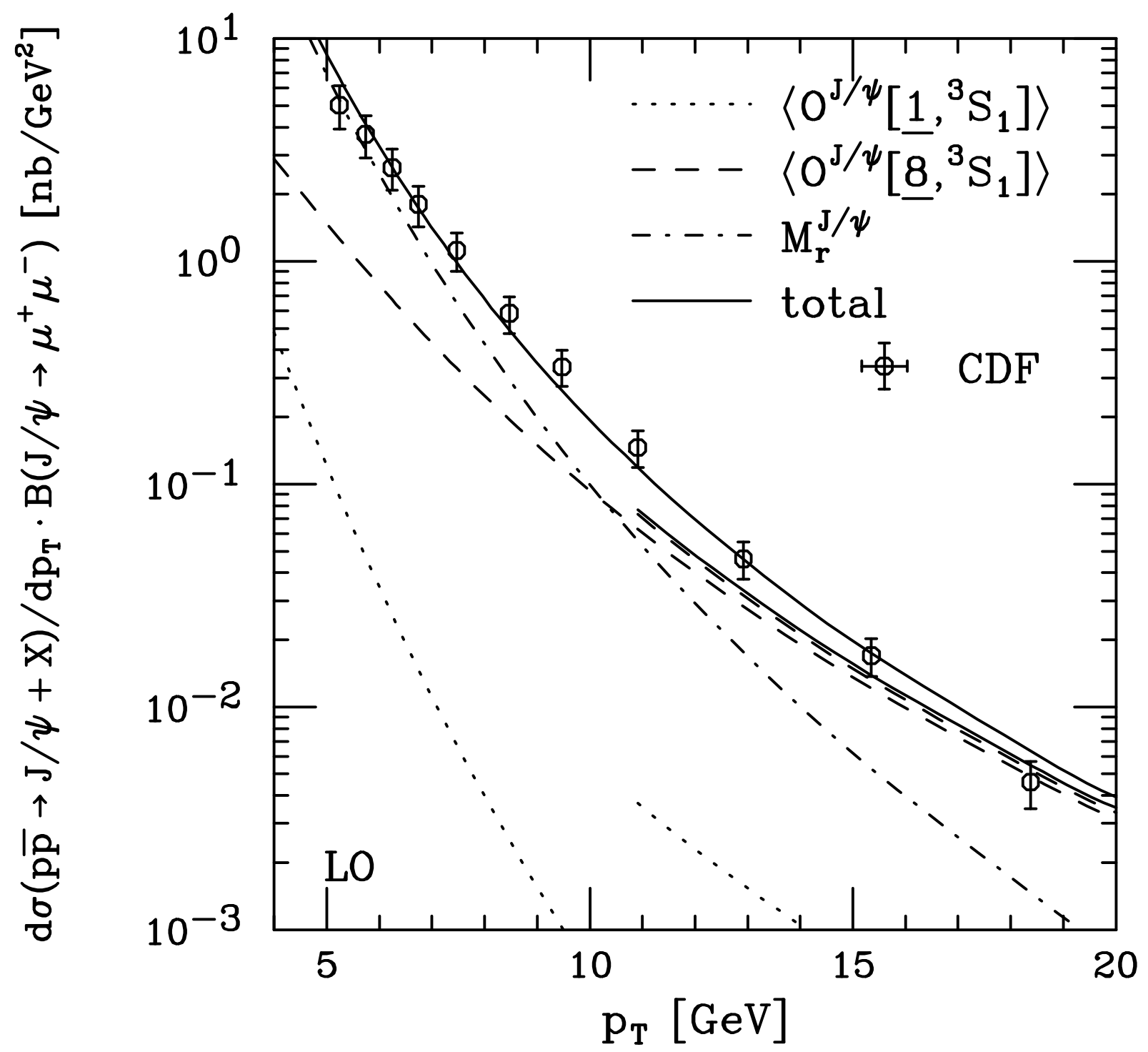

Fig. 1a 


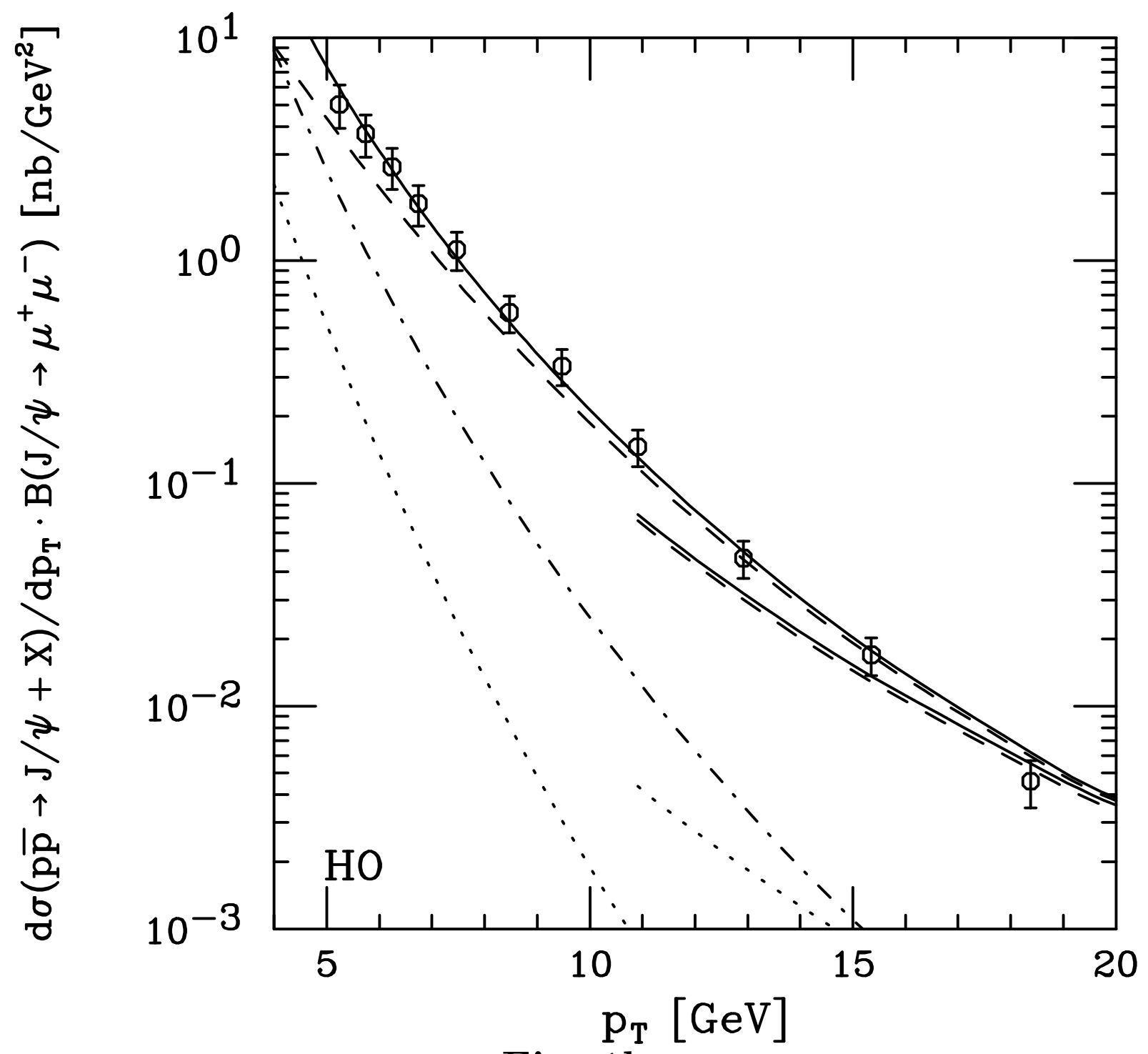

Fig. 1b 


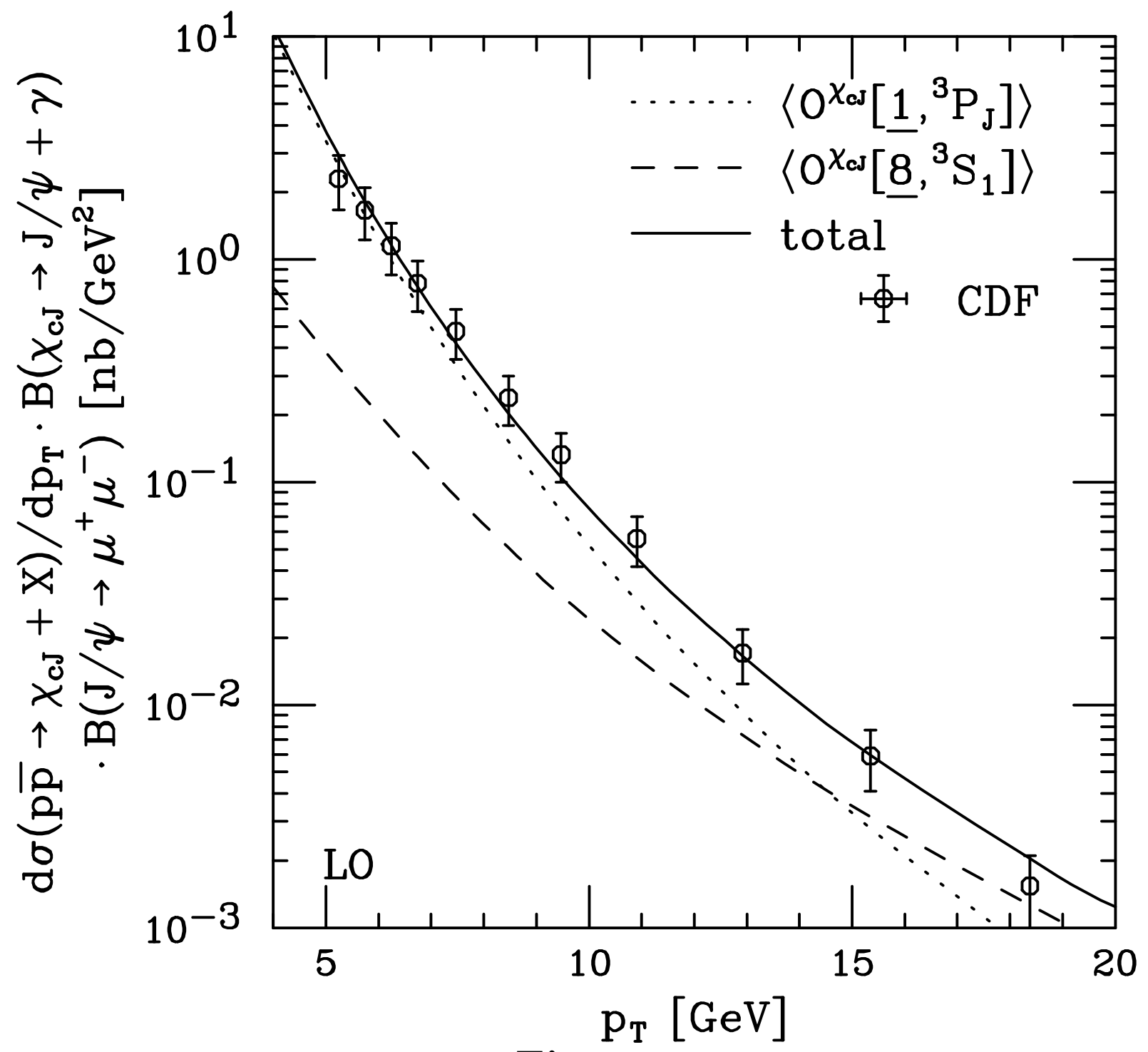

Fig. 2 


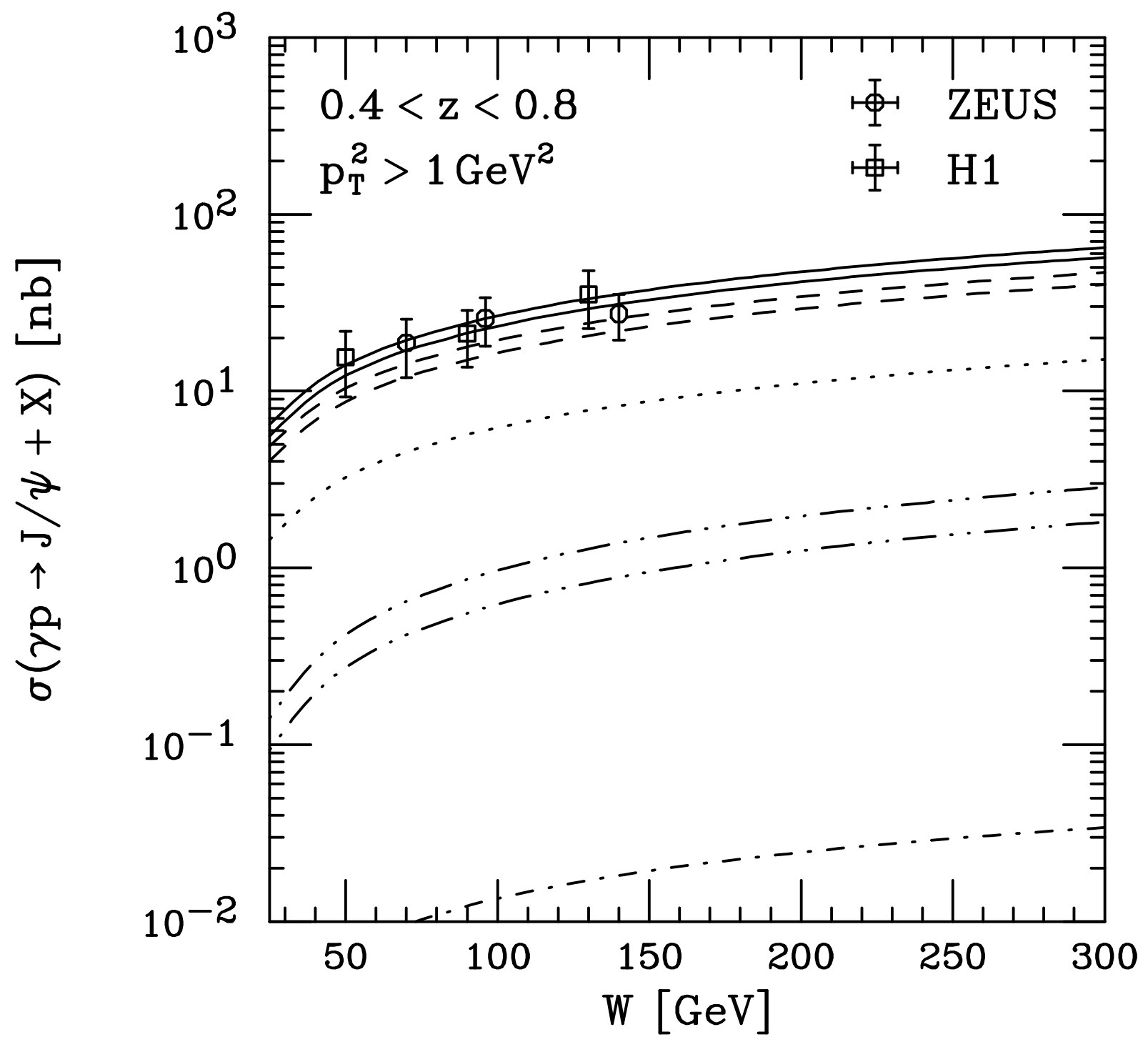

Fig. 3a 


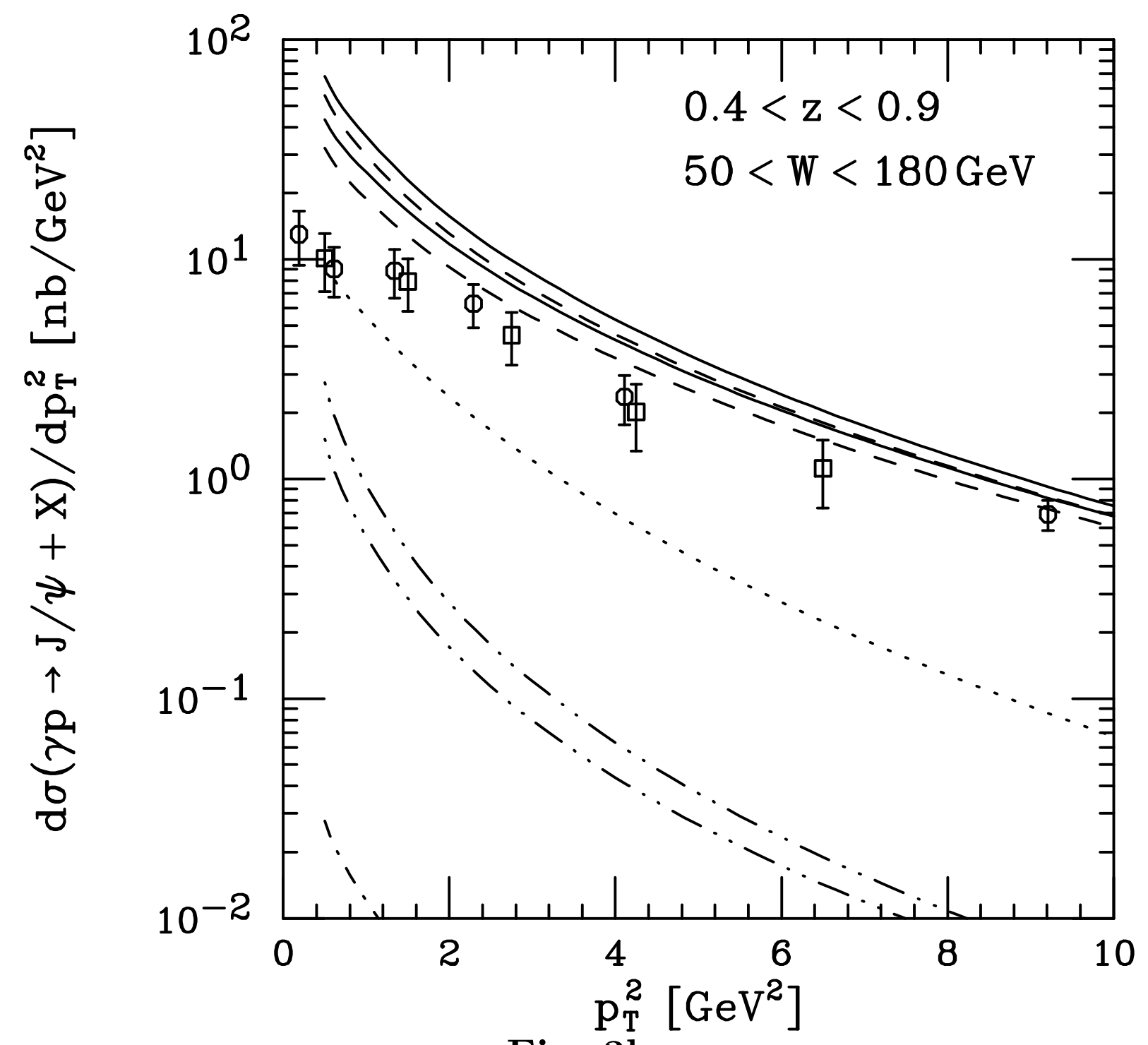

Fig. $3 b$ 


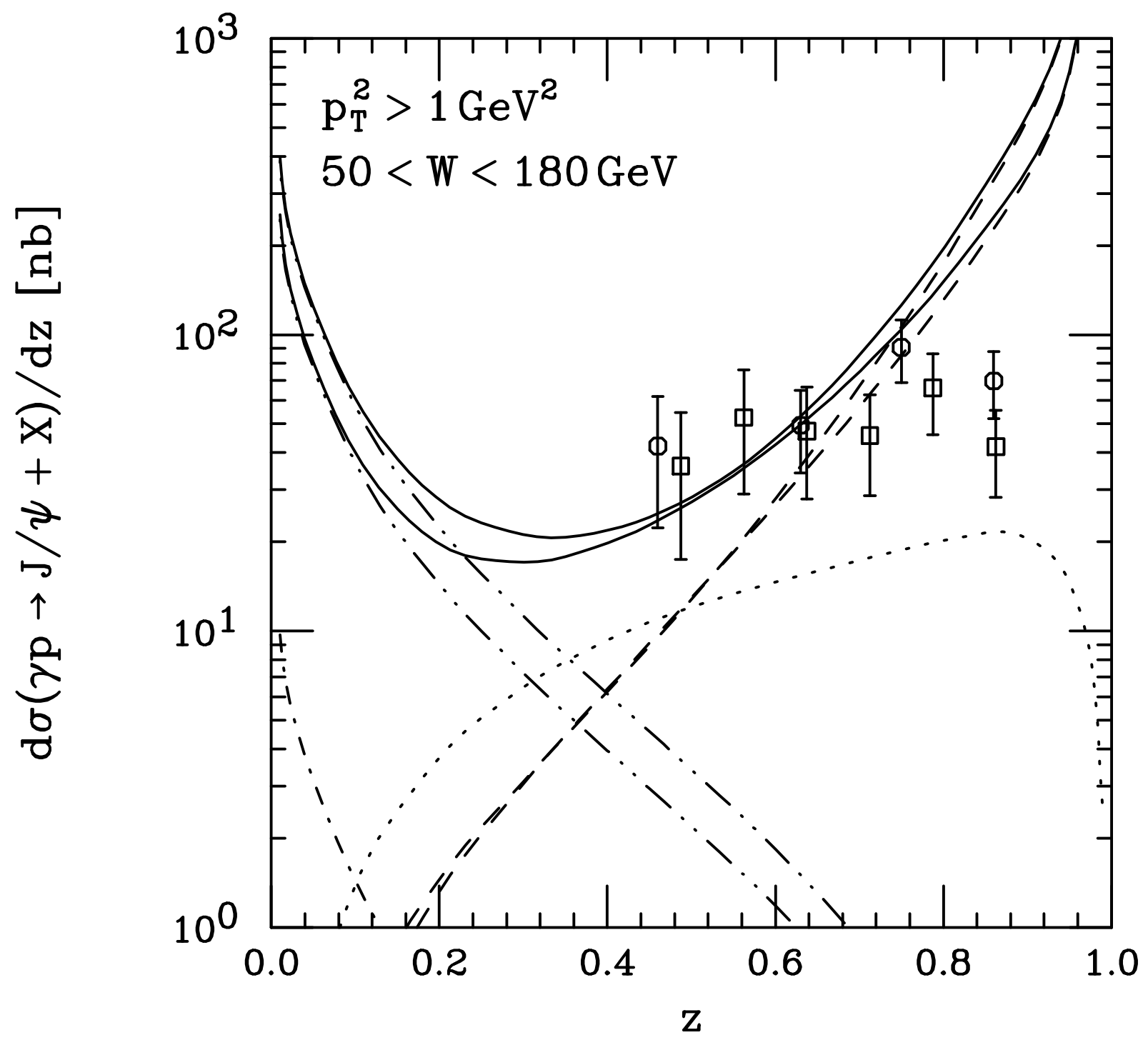

Fig. 3c 


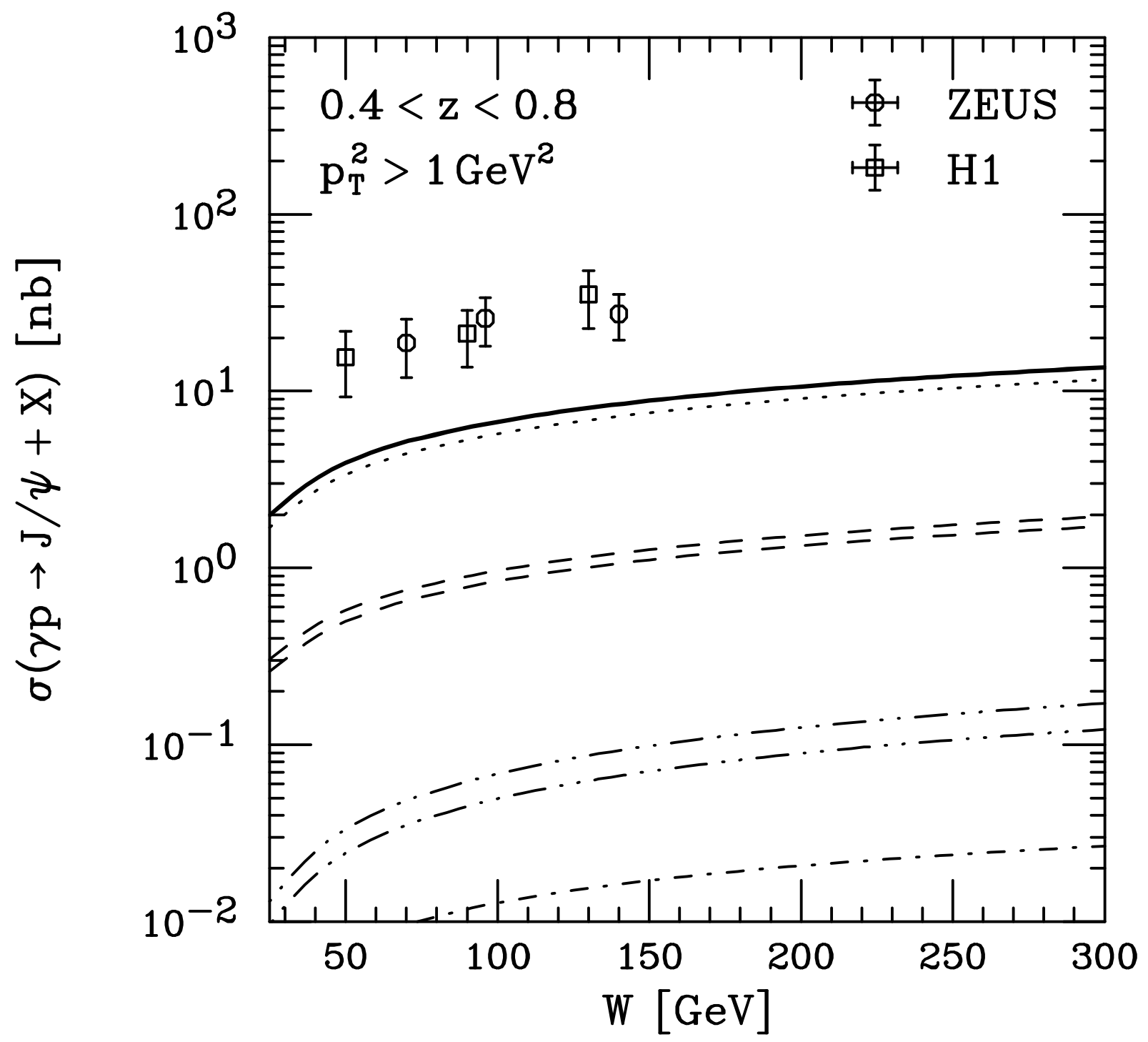

Fig. 4a 


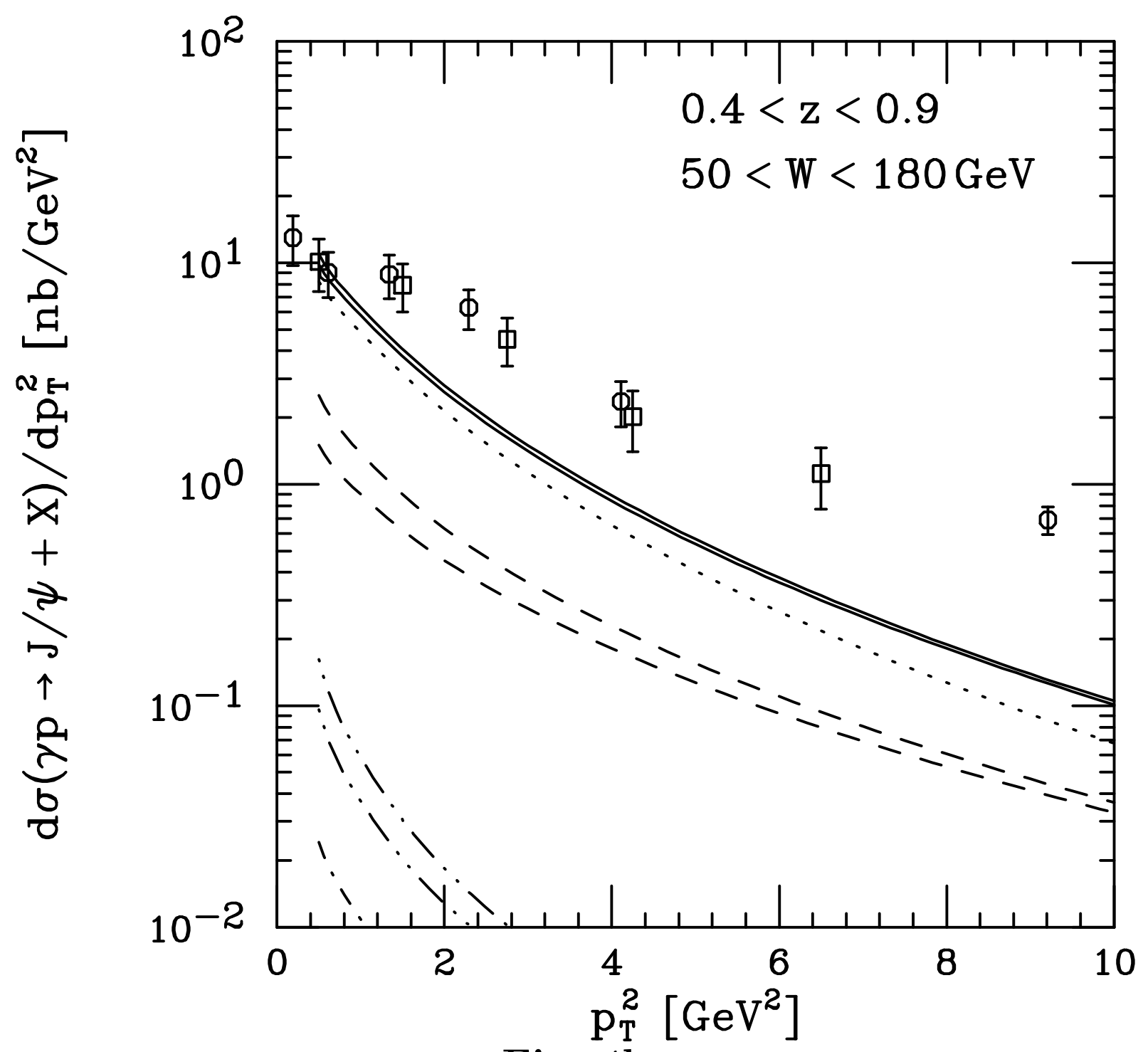

Fig. $4 \mathrm{~b}$ 


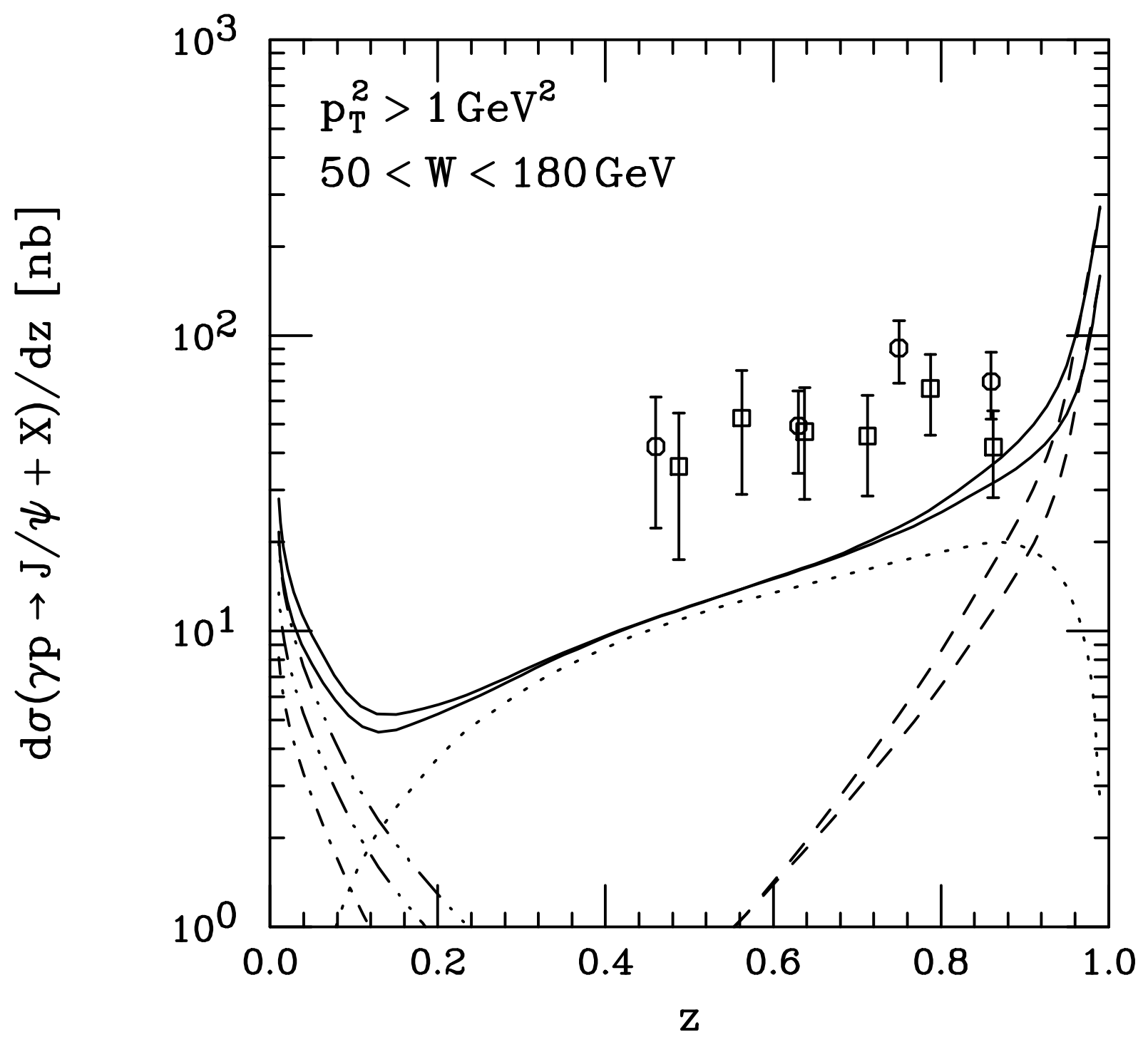

Fig. 4c 


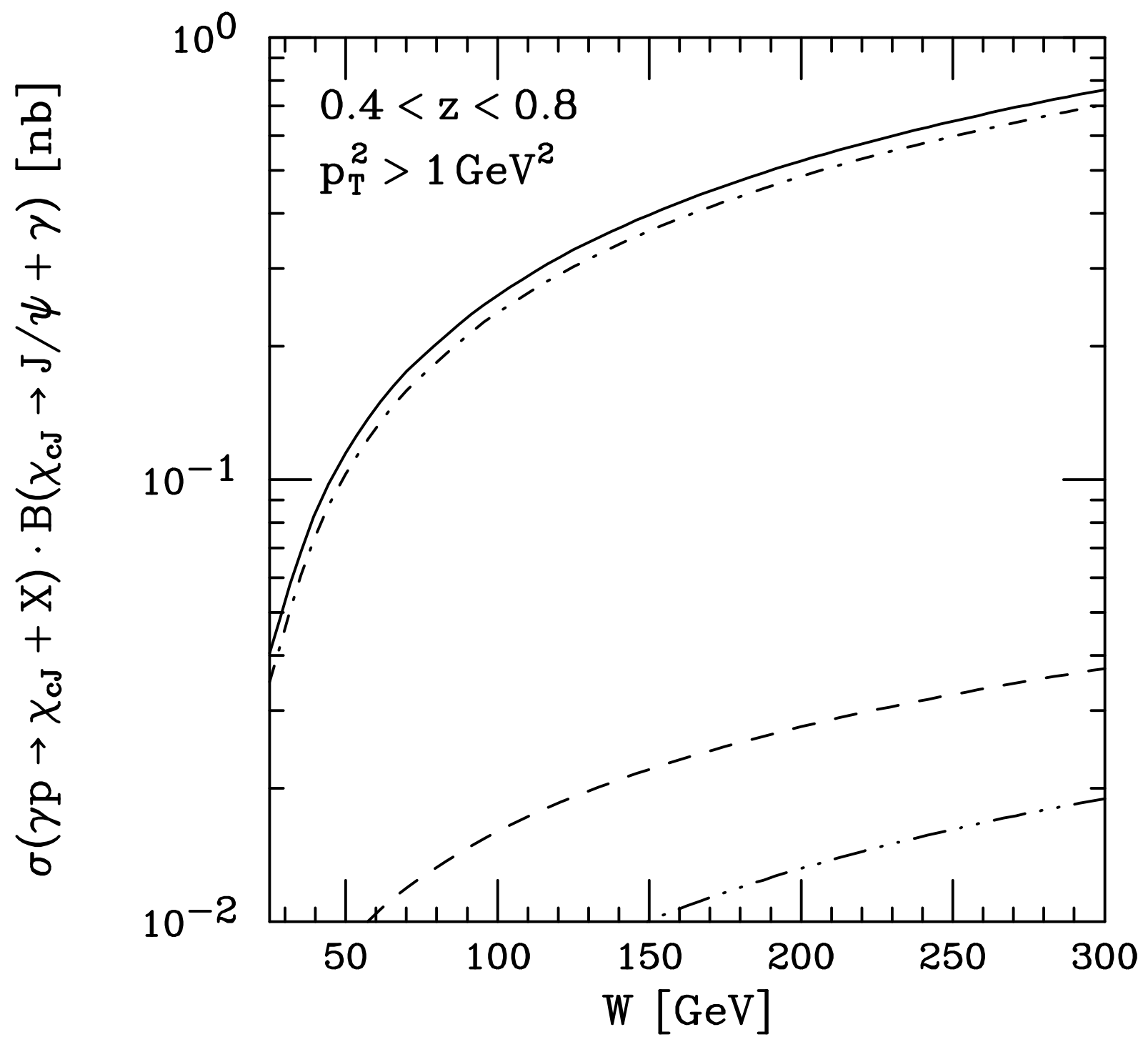

Fig. 5a 


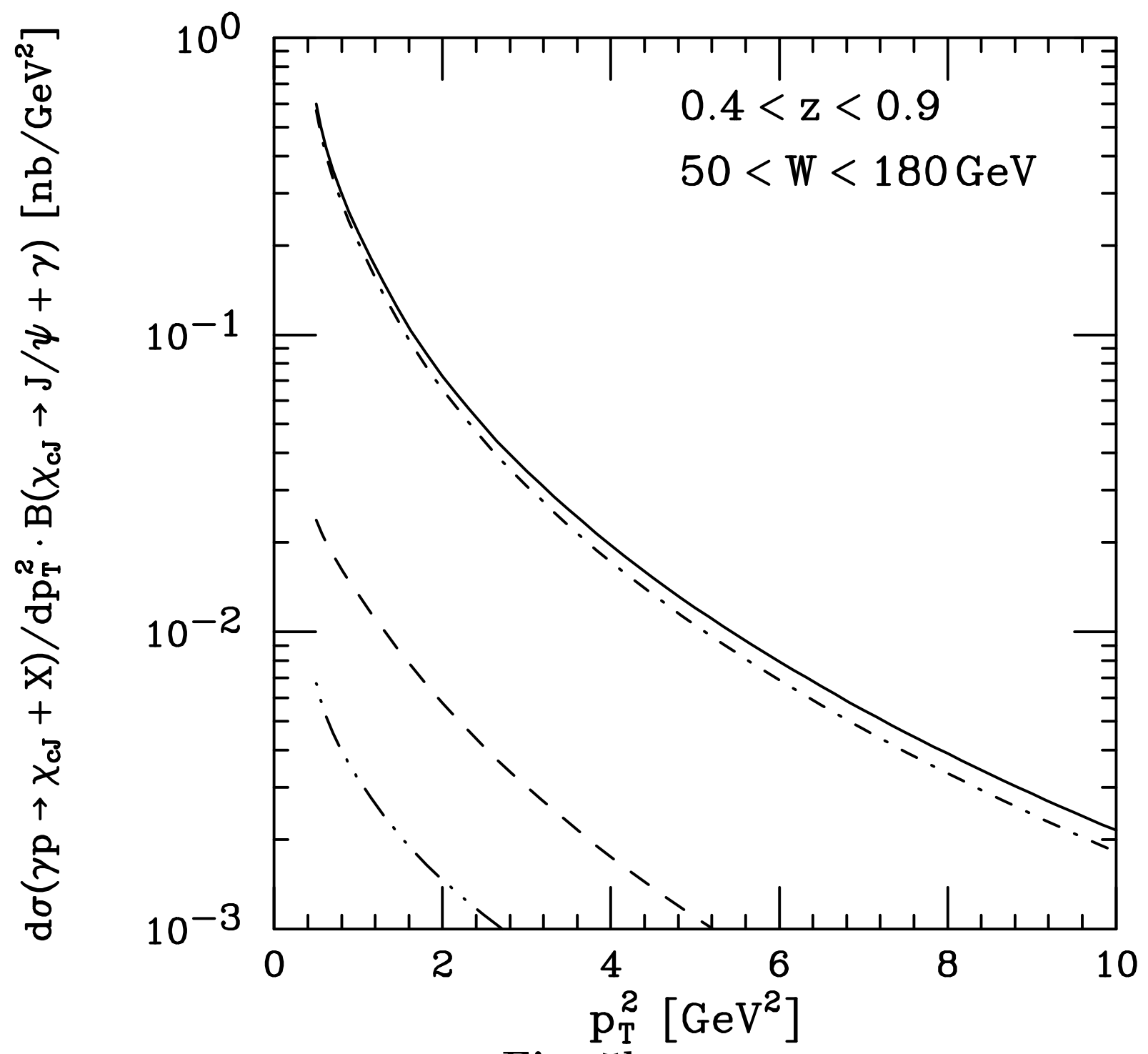

Fig. 5b 


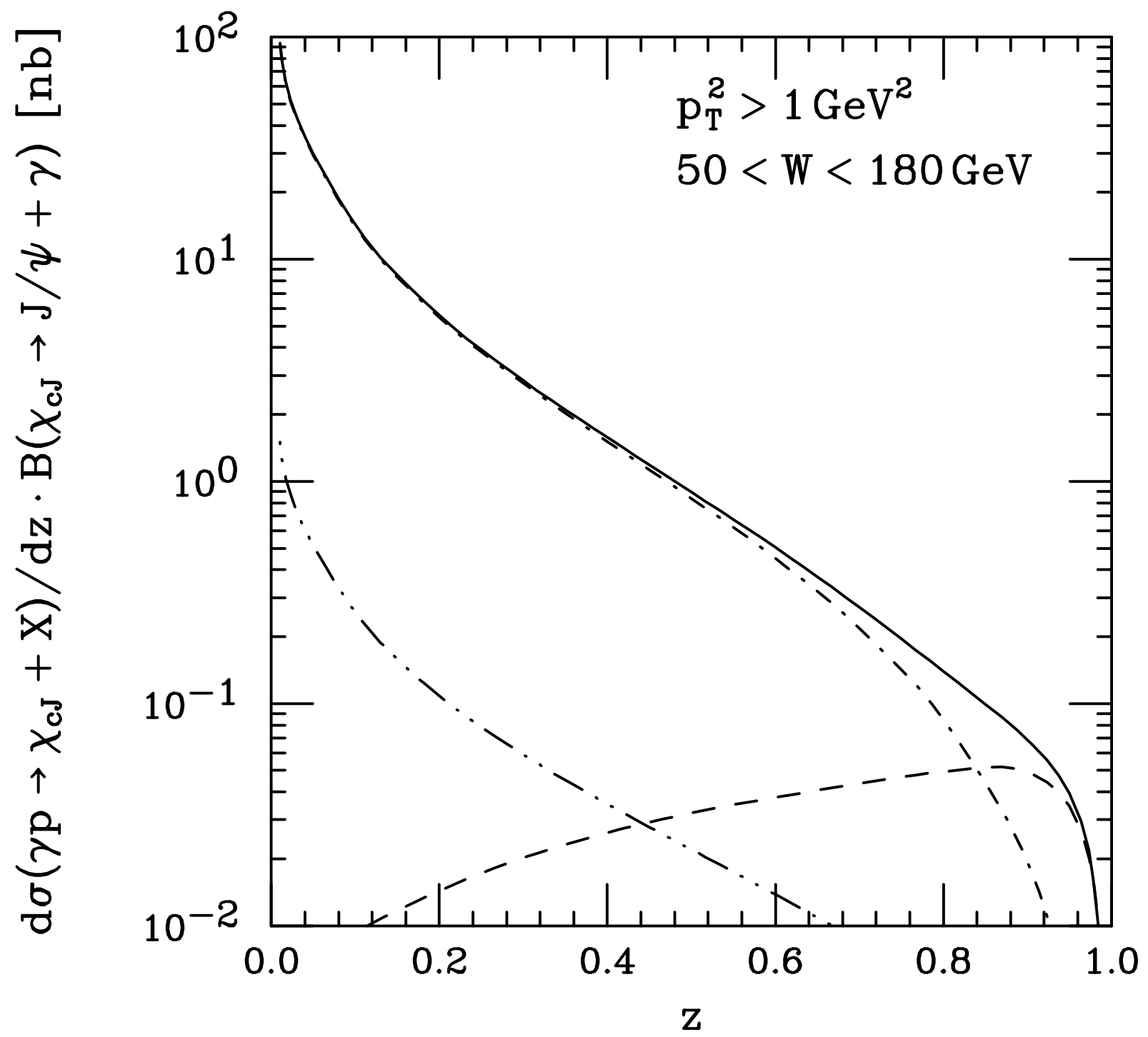

Fig. 5c 